\title{
Commentary: Keep your ion the urine: A new way to predict postoperative acute kidney injury?
}

\author{
Alexander Zarbock, MD, ${ }^{\mathrm{a}}$ and Daniel T. Engelman, $\mathrm{MD}^{\mathrm{b}}$
}

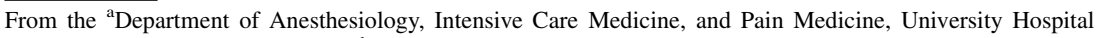
Münster, Münster, Germany; and ${ }^{\mathrm{b}}$ Department of Surgery, Baystate Medical Center, Springfield, Mass.

Disclosures: Dr Zarbock receives speaking honoraria from Baxter, Astute Medical, Fresenius, Ratiopharm, Amomed, bioMerieux, and Braun and has received funding support from German Research Foundation, GIF, BMBF, Fresenius, Astellas, and Astute Medical. Dr Engelman consults for bioMerieux, Edwards Lifesciences, and Zimmer-Biomet.

Received for publication Feb 26, 2019; accepted for publication Feb 26, 2019; available ahead of print April 11, 2019.

Address for reprints: Alexander Zarbock, MD, Department of Anesthesiology, Intensive Care Medicine, and Pain Medicine, University Hospital Münster, Albert-Schweitzer-Campus 1, Gebäude A1, 48149 Münster, Germany (E-mail: zarbock@uni-muenster.de).

J Thorac Cardiovasc Surg 2020;159:928-9

$0022-5223 / \$ 36.00$

Copyright $@ 2019$ by The American Association for Thoracic Surgery

https://doi.org/10.1016/j.jtcvs.2019.02.111
}

Acute kidney injury (AKI) is a common complication after cardiac surgery and influences morbidity and mortality. The incidence of cardiac-surgery-associated AKI ranges from $10 \%$ to $30 \%$, depending on the complexity of the procedure. $^{1}$

The diagnosis and staging of AKI according to the Kidney Disease: Improving Global Outcomes guidelines is based on changes in serum creatinine level and/or urine output, reflecting a change in kidney function. ${ }^{2}$ However, these functional biomarkers have several limitations. In contrast, new damage biomarkers allow a diagnosis to be made earlier and allow kidney injury to be diagnosed even in the absence of concurrent or subsequent dysfunction. $^{3}$ A biomarker-based approach was recently utilized in the Prevention of Cardiac Surgery-associated AKI by Implementing the Kidney Disease: Improving Global Outcomes Guidelines in High Risk Patients Identified by Biomarkers randomized controlled trial. ${ }^{4}$ This demonstrated that implementing a bundle of supportive measures in high-risk patients identified by urinary biomarkers significantly reduced the occurrence of AKI after cardiac surgery. ${ }^{4}$

Recent studies suggest that ion homeostasis is involved in the pathophysiology of AKI. Shen and colleagues ${ }^{5}$ present the results of an observational trial investigating whether the composition of urinary ions can predict AKI after cardiac surgery. By performing urinary ionomic analyses, the authors identified that the concentrations of most ions, independent of kidney function, increased in the urine after cardiac surgery. In particular, the increase in select ions 2 hours after cardiac surgery was higher in patients who subsequently developed AKI. Based on these differences, the authors developed a urinary ion index (UII) to predict cardiac surgery-associated AKI, need for renal replacement therapy, and 30-day mortality.

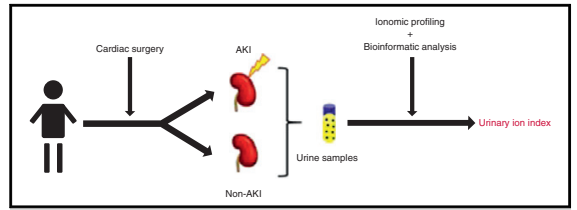

Study flow of the observational trial in which the urinary ion index was developed.

\section{Central Message}

Profiling ions in the urine immediately after cardiac surgery may be a new method to predict the development of cardiac-surgery associated $\mathrm{AKI}$, need for renal replacement therapy, and mortality.

See Article page 918.
Although the approach and methods the authors used are very novel and innovative, the study is not without several limitations. A major limitation of the study is the limited generalizability because the authors specifically excluded patients with reduced kidney function. However, because many patients undergoing cardiac surgery have reduced kidney function and ion homeostasis is usually disturbed in patients with chronic kidney disease, ${ }^{6}$ the utility of the test is limited by these factors. Furthermore, the use of cardiopulmonary bypass was different between the AKI and non-AKI groups. Because the transfusion rate of blood products is higher in on-pump surgery, ${ }^{7}$ this may have influenced the results. The transfusion of blood products also influences iron levels, which is the main driver of the UII. In addition, the use of cardiopulmonary bypass induces hemolysis, which can influence ion levels. Based on the aforementioned limitations and the small sample size of the study, the UII will need to be validated in future, larger, heterogeneous patient populations.

\section{References}

1. Lassnigg A, Schmidlin D, Mouhieddine M, Bachmann LM, Druml W, Bauer P, et al. Minimal changes of serum creatinine predict prognosis in patients after cardiothoracic surgery: a prospective cohort study. J Am Soc Nephrol. 2004;15: 1597-605.

2. KDIGO clinical practice guideline for acute kidney injury. Kidney Int. 2012;2: $1-138$.

3. Ronco C, Kellum JA, Haase M. Subclinical AKI is still AKI. Crit Care. 2012;16: 313. 
4. Meersch M, Schmidt C, Hoffmeier A, Van Aken H, Wempe C, Gerss J, et al. Prevention of cardiac surgery-associated AKI by implementing the KDIGO guidelines in high risk patients identified by biomarkers: the PrevAKI randomized controlled trial. Intensive Care Med. 2017;43:1551-61.

5. Shen Z, Lin J, Teng J, Zhuang Y, Zhan H, Wang C, et al. Association of urinary ionomic profiles and acute kidney injury and mortality in patients after cardiac surgery. J Thorac Cardiovasc Surg. 2020;159:918-26.e5.
6. Hasegawa H, Nagano N, Urakawa I, Yamazaki Y, Iijima K, Fujita T, et al. Direc evidence for a causative role of FGF23 in the abnormal renal phosphate handling and vitamin D metabolism in rats with early-stage chronic kidney disease. Kidney Int. 2010;78:975-80.

7. Lamy A, Devereaux PJ, Prabhakaran D, Taggart DP, Hu S, Paolasso E, et al. Offpump or on-pump coronary-artery bypass grafting at 30 days. N Engl J Med. 2012; 366:1489-97. 